\title{
Aspects of the psychology of spending
}

\author{
SOTOHIRO KOJIMA \\ Department of Psycholog., Faculty of Letters, Doshisha Unitersity, Kamigyo-ku, Kyoto 602
}

YASUHISA HAMA

Department of Behavioral Science, Hokkaido Lniversity, Kita-ku, Sapporo 060

When a consumer purchases a commodity, the expenditure may or may not be accompanied by grudging. The purpose of this survey was to evaluate the difference of " the psychology of spending" by using the three scales of: (1) psychological grudging accompanying expenditure, (2) necessity of the commodity, and (3) popularity of the commodity. Two hundred and seventeen housewives were asked to evaluate 50 commodities by the three scales on six levels, Factor analysis of ratings were carried out for each scale. The seven common "psychologies of spending "-so called "psychological moneybags "-among three scales were found. These were (1) daily necessities, (2) a small luxury, (3) culture and education, (4) personal fortune, (5) security, (6) feminine articles, and (7) eating out.

Key words: consumer psychology, marketing, commodity classification, economic psychology, expenditure.

With few exceptions, economic activities in contemporary societies are founded on money as the basic medium. Consider the consumer: his consumer activities are basically an economic operation whereby the consumer supplies money in order to obtain corresponding commodities or services. When this consumer purchases a commodity or service in reality, the money is supplied from a so-called " moneybag". In some cases this does not necessarily mean the wallet, but may be in the form of a credit card, check, or a transfer of money from a bank account. However, these differences are of no importance in this survey. The overall idea enveloping this, including money itself, checks and deposits, will be broadly defined as " the spending money". Along these lines, there is really only one definition of spending from which the payment is made and this spending becomes the contact point between the consumer and the supplier. For example, considered the phenomenon, if the expenditure is $\$ 50.00$,--there is $\$ 50.00$ less-regardless of what the money was spent for. However, the same expenditure of $\$ 50.00$ brings about a dif- ferent psychological meaning, depending upon whether it was spent on rice or "matsutake"." It can be presumed that the $\$ 50.00$ for rice would be spent with relatively littlc objection as it is a necessary expense, whereas spending the same $\$ 50.00$ on matsutake would be a radical decision.

As can be seen from such an example, it is difficult to conceive an actual act of purchasing being limited to one single interpretation based upon one definition of spending. A more appropriate interpretation would be the existence of several invisible "psychologies of spending" in the background which operate prior to the act, ultimately resulting in the tightening or the loosening of the strings around the real "moneybag". In the case of the choice between the rice and matstutake, the rice is paid for from the spending psychology of necessary purchases, while the "matsutake" is paid for from the spend-

1 Matsutake is a mushroom, Amillaria matsudake, very fragrant and valued by the Japanese as a seasonal delicacy; extremely expensive due to high demand and very limited supply. 
ing psychology for " a small luxury", resulting, therefore, in a different degree of reluctance felt in the expenditure of the same $\$ 50.00$. A generalization of such phenomena could be thought of as a problem of "intra-individual spending psychology" of one consumer, due to the "difference in the spending psychology" of the consumer, related to the different commodities.

In addition, even when the $\$ 50.00$ "matsutake" is purchased, the grudging of the expenditure will vary depending upon whether it had been purchased as a gift for someone special, or for consumption by the family at home. This is a problem of "the difference in the spending psychology for the same commodity": the difference in the grudging felt by purchasing the same commodity depending upon the individual circumstances and the time, place, and occasion. This phenomenon of variability in the psychological grudging accompanying expenditure can be seen in everyday life as well. Especially in the case of Japanese people, there seems to be a tendency of becoming generous with money on trips so that even those who count pennies at the grocers find it not too difficult to pay for the relatively expensive oranges sold on trains. Although this problem of the "intra-individual spending psychology for the same commodity" is an interesting theme in considering consumer activities, the " intra-individual spending psychology for the different commodities" will be considered collectively, on a mass level in this survey. In other words, our purpose was to find out how each commodity was positioned within the value structure of the consumers by utilizing the concept of the "psychology of spending". Thus, the "psychology of spending" is an alternate way of expressing the existence of the various rulers (scales) for value judgment of commodity purchasing by the consumers.

The types of "spending psychology", including the ones mentioned above, are as follows:

1. Difference in "intra-individual spending psychology" for the different commodities. (mentioned above)

2. Difference in "intra-individual spending psychology" for the same commodity. (mentioned above)

3. Difference in "inter-individual spending psychology" for the different commodities.

This category expresses the difference in the commodities valued by different people. For example, there are those who would, only with reservations, spend $\$ 25.00$ on a meal, but would gladly spend the same $\$ 25.00$ on books.

4. Difference in the "inter-individual spending psychology" for the same commodity.

This shows the difference in the value scale for the same commodity between different people, as, for example, the difference between those who would think of the purchase of a video-tape recorder as extremely valuable and those who would feel that such an expenditure is wasteful and unnecessary.

This concept of the "psychology of spending " was first introduced by Kojima (1964, 1972). Since then, the Asahi Shinbun (1972) has conducted mass surveys on the first type of problem mentioned previously with the "psychological grudging accompanying expenditure" scale. The Asahi Shinbun found seven main "psychologies of spending ". This result was compiled and published by Tanaka and Kitade (1974).

In our present study, the above-mentioned points were taken into consideration, and the scales of "necessity" and "popularity" of the commodities were added to the "psychological grudging accompanying expenditure" scale, in examining the problem of the first type of "spending psychology".

If a "spending psychology" can be extructed from the consumer's recognition of 
commodities by each of these scales, much light will be shed on the understanding of consumer's purchasing behavior.

\section{Method}

In February, 1979, 217 housewives in the Kansai area were asked to evaluate the 50 commodities for their "psychological grudging accompanying expenditure", " necessity" for living in society, and "popularity" at this point in time, on six levels. They were: extremely necessary ( 6 points), moderately necessary (5 points), somewhat necessary (4 points), probably not necessary ( 3 points), not really necessary ( 2 points), and not necessary at all (1 point). These 50 items were chosen referring to those used in the Asahi Shinbun survey (1972), with several new commodities added to include as many fields as possible.

\section{Results and Discussion}

\section{Preliminary Compilation}

Table 1 shows the top 10 commodities which have high secores in the three scales. As can be seen from the table, " necessity" and "popularity" show extremely similar results. In particular, those with a high level recognition for "necessity" show a tendency to have a high level recognition for "popularity" as well. However, the "psychological grudging accompanying expenditure" for such commodities is not necessarily small, but shows rather that the grudging is smaller with not-so-necessary but cheap commodities, such as chewing gum and chocolate. This is due to the price itself, rather than to the logical, conceptional idea of the "necessity" or "popularity" of the commodities.

The extremely positive commodities which had a high level of " necessity" and "popularity" with correspondingly small "psychological grudging accompanying expenditure", were the three com- moditics: refrigerator and washing machine-representing daily necessities-and lipstick. The emergence of lipstick among the top three commodities must have been due to the fact that the subjects of the survey were women (housewives).

In addition, those commodities which have a large value in the "psychological grudging accompanying expenditure" have a tendency of being strongly influenced by high prices, although they are also related to the low values of "necessity" and "popularity". Negative commodities which are low in both " necessity" and "popularity" and also have high "psychological grudging accompanying expenditures" were the following four commodities: land for a summer house, dishwashers, video-tape recorders and sound movie cameras.

\section{Factor Analysis}

It was possible to survey the recognition pattern by the three scales and their general relation to each other from the PRELIMINARY COMPILATION. Then, in order to extract the "psychology of spending ", factor analysis was carried out each item in all three scales. As a result, nine factors for the "psychological grudging accompanying expenditure", 11 for "necessity", and nine for "popularity" were extracted. Tables 2, 3, and 4 show the representative commodities of each factor in each of the scales from the largest factor loading.

1. Seven "spending psychologies". The seven "spending psychology factors", or the factors commonly extracted from the three scales were: "daily necessities", "a small luxury", "culture and education", "personal fortune", "security", "feminine articles" and " eating out". Table 5 shows the three scales corresponding with the seven common factors extracted from them. These seven "spending psychology factors" may be regarded to be extremely important in considering purchasing behavior. With this as a clue, we 
Table 1

Top 10 commodities of "psychological grudging accompanying expenditure", "necessity", and "popularity"

\begin{tabular}{|c|c|c|c|}
\hline 1. Not & grudging to pay & $\begin{array}{l}\text { Recognized } \\
\text { for necessity }\end{array}$ & $\begin{array}{l}\text { Recognized } \\
\text { for popularity }\end{array}$ \\
\hline (1) & Eye drops & & \\
\hline$(2)$ & Chewing gum & & \\
\hline (3) & Lipstick & * & $*$ \\
\hline (4) & Washing machine & * & * \\
\hline (5) & Chocolate & & \\
\hline (6) & $\begin{array}{l}\text { One's own meal expenses when one has } \\
\text { eaten with friends }\end{array}$ & & \\
\hline$(7)$ & Aspirin & & \\
\hline$(8)$ & Coffee break with friends & & \\
\hline (9) & Refrigerator & * & * \\
\hline$(10)$ & Teatime cakes & & \\
\hline \multicolumn{2}{|c|}{ 2. Recognized for necessity } & $\begin{array}{l}\text { Not grudging } \\
\text { to pay }\end{array}$ & $\begin{array}{l}\text { Recognized for } \\
\text { popularity }\end{array}$ \\
\hline (1) & Refrigerator & * & * \\
\hline$(2)$ & Washing machine & * & * \\
\hline (3) & Wardrobe & & $*$ \\
\hline (4) & Husband's monthly life insurance premium & & * \\
\hline (5) & Fire and house insurance & & * \\
\hline (6) & Color television & & * \\
\hline$(7)$ & Lipstick & * & * \\
\hline$(8)$ & Handbag & & $*$ \\
\hline (9) & Car insurance & & $*$ \\
\hline$(10)$ & Encyclopedia & & \\
\hline \multicolumn{2}{|c|}{ 3. Grudging to pay } & $\begin{array}{l}\text { Not recognized } \\
\text { for necessity }\end{array}$ & $\begin{array}{l}\text { Not recognized } \\
\text { for popularity }\end{array}$ \\
\hline (1) & Land for summer house & $*$ & $*$ \\
\hline (2) & Dishwasher & * & $*$ \\
\hline (3) & Land & & $*$ \\
\hline (4) & Video-tape recorder for $\mathrm{T} . \mathrm{V}$. & $*$ & $*$ \\
\hline (5) & Apartment house & & \\
\hline (6) & Sound movie camera & * & $*$ \\
\hline (7) & Piano & & \\
\hline (8) & House & & \\
\hline (9) & Gentral heating & & $*$ \\
\hline$(10)$ & Gar & & \\
\hline
\end{tabular}

would like to discuss how the commodities are ranked according to the "spending psychology".

(I) Daily necessities. In all three scales, commodities such as refrigerators, wardrobes, and washing machines are clustered in this factor. These commodities were those which were regarded as being extremely positive in the "preliminary compilation" and can be designated as essential for daily life. This result suggests that color television has been recognized 
Table 2

Results of the factor analysis based on "psychological grudging accompanying expenditure" scale

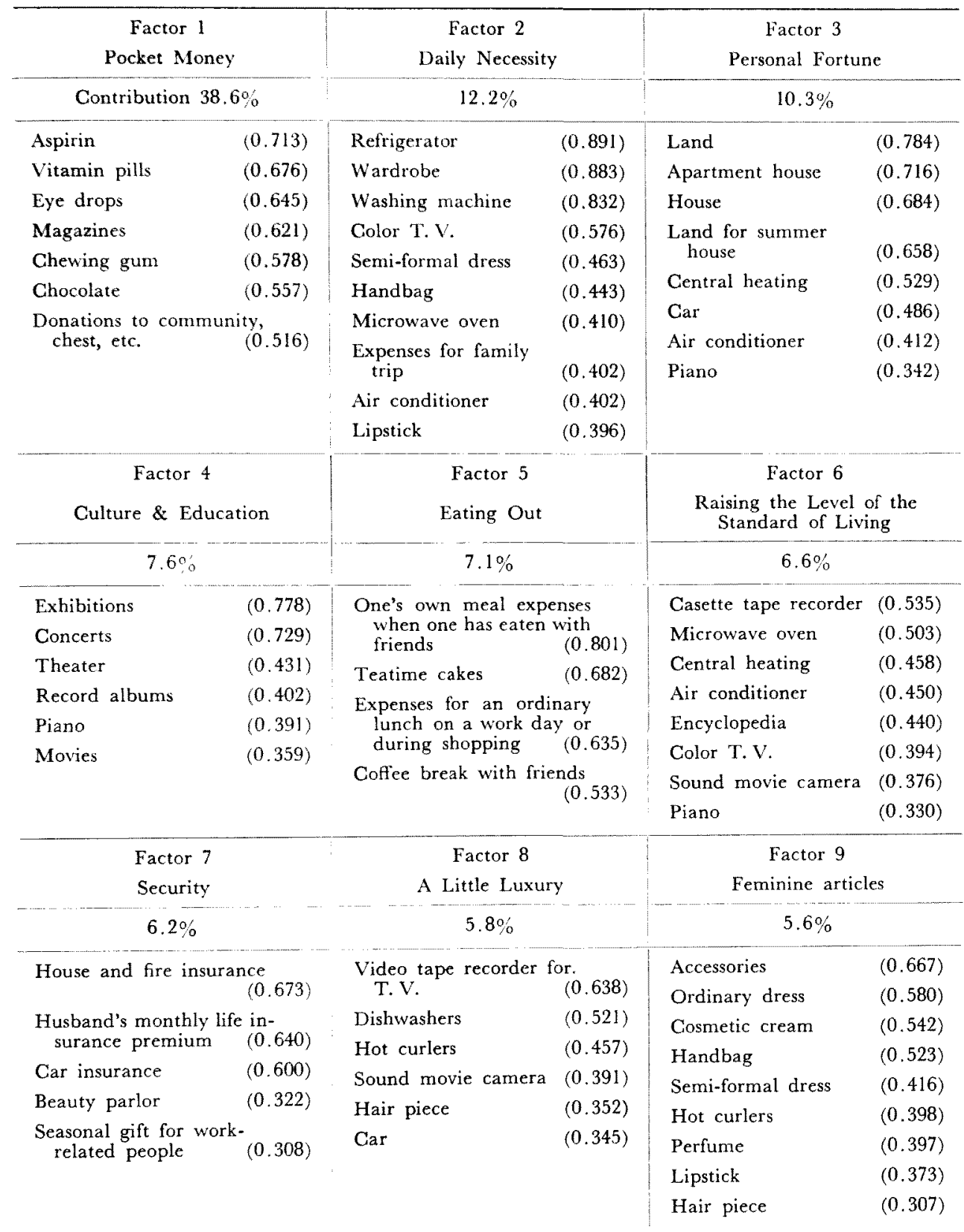

as an important thing, showing the progressive change in the attitude of the consumer.
(2) A small luxury. The items which are purchased out of this "moneybag" are clustered around those which are eval- 
Table 3

Results of the factor analysis based on "necessity" scale

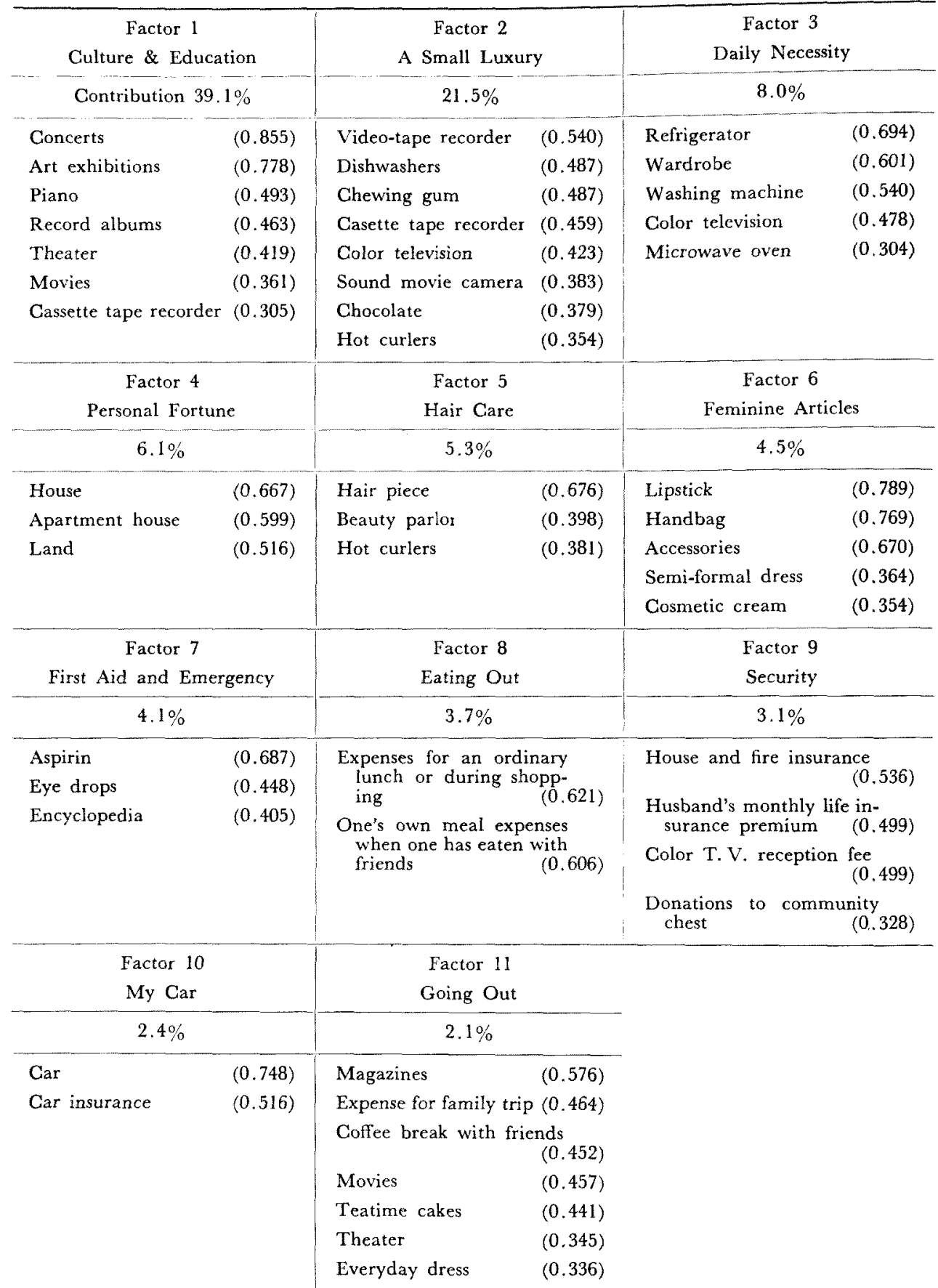


Table 4

Results of the factor analysis based on "popularity" scale

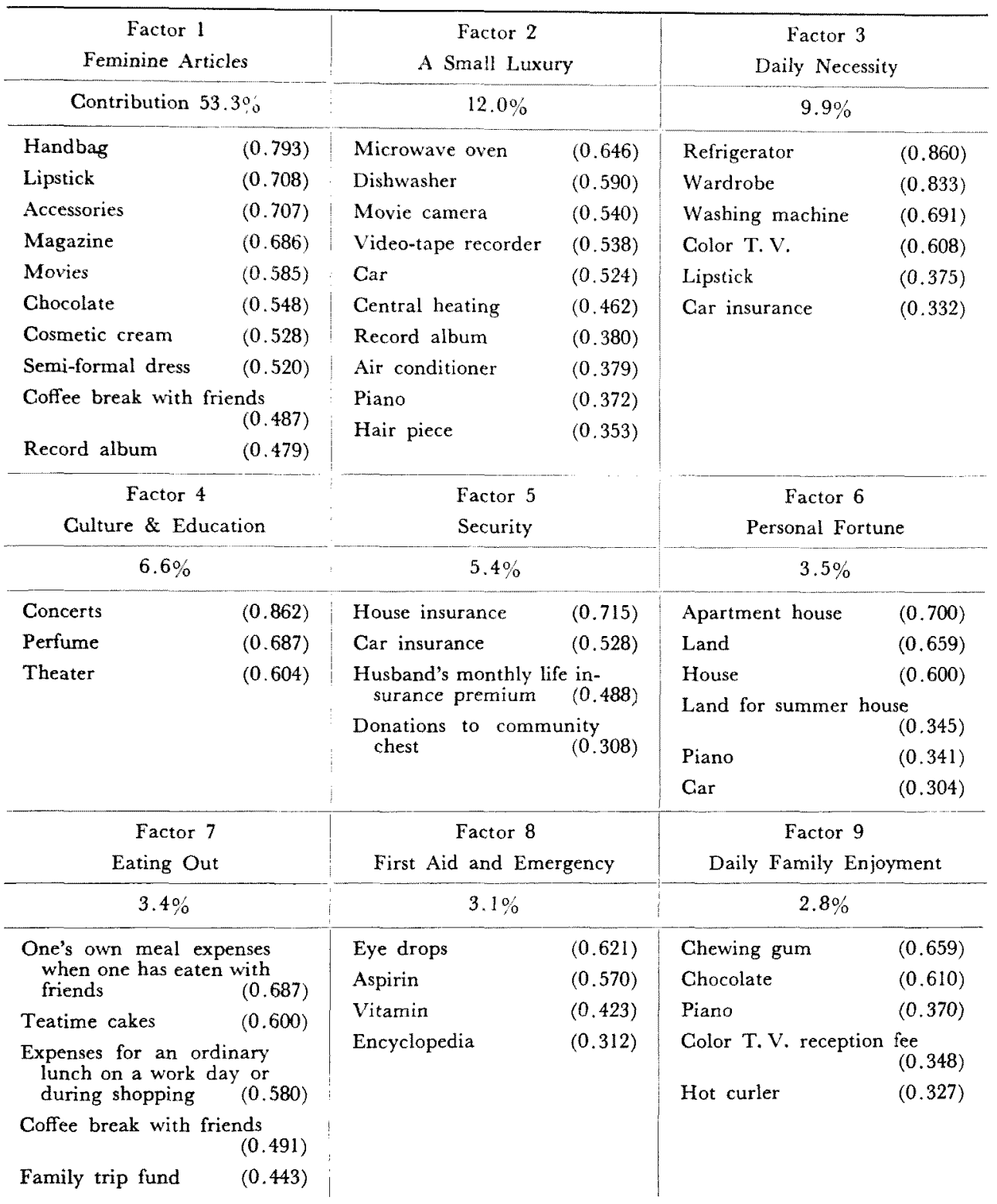

uated negatively in the "simple compilation" such as: television, video-tape recorder, dishwasher and sound camera. However, those commodities, considered as extremely luxurious at this point, will become daily necessities in the future, as in the case of color television with its rapid popularization. The image of dishwashers being expensive and not useful seems to be firmly grounded, but commodities such 
Table 5

Factor correspondence of the three scales

\begin{tabular}{|c|c|c|c|}
\hline \multirow[b]{2}{*}{ Factor } & \multicolumn{3}{|c|}{ Scale } \\
\hline & Necessity & Popularity & $\begin{array}{l}\text { Psychological } \\
\text { grudging }\end{array}$ \\
\hline Daily Necessity & * & $*$ & $*$ \\
\hline A Small Luxury & * & $*$ & * \\
\hline Gulture and Education & * & $*$ & $*$ \\
\hline Personal Fortune & * & * & * \\
\hline Security & * & $*$ & $*$ \\
\hline Feminine Articles & * & $*$ & $*$ \\
\hline Hair Care & * & & \\
\hline Eating Out & * & $*$ & * \\
\hline Going Out & * & & \\
\hline First Aid and Emergency Equipment & * & * & \\
\hline My Car & $*$ & & \\
\hline Daily Family Enjoyment & & * & \\
\hline Pocket Money & & & * \\
\hline Raising the Level of the Standard of Living & & & $*$ \\
\hline
\end{tabular}

as the video-tape recorder for television and the sound movie camera are emerging as luxury factor.

When the scales are compared, it is noted that the factor "a small luxury" partially includes the factor of "raising the level of the standard of living " in the popularity scale.

Also, it is interesting to note that color television, which seemed to be completely a part of the " daily necessity", is clustered in the factor of "a small luxury" in the "necessity" scale. Perhaps this shows a lingering notion of the psychological discrimination between color television and washing machines.

(3) Culture and education. For this factor, commodities with hobby-like aspects, especially related to visual and audio articles are clustered. The motivation for going to a concert or listening to a record album is basically the same, and their effects may be similar as well. In this case, the concert ticket and the cost of the record are both paid for from the same "psychology of spending".

(4) Personal fortune. The main expen- ditures of this factor are related to real estate. The categorization of household appliances such as central heating and air conditioning in this factor is easily comprehensible. But the interesting point is the inclusion of cars and pianos. Of course, cars and pianos are expensive commodities, however this is not the only reason for including them in "personal fortune". So, this aspect cannot be ignored in considering the advertising of these commodities. In the case of cars, appealing too much to the property value may bring a negative effect to lengthen the car changing. However, with this in mind, an appeal such as "your private mansion" may have great effect.

(5) Security. In this case, various kinds of insurance form the core. Community chest is also similar to insurance in that the money collected is given to disaster victims. Even the television reception fee, beauty parlor and "oseibo" (the year-end gifts given to special people who, supposedly, have done something for you) bills all seem to come out of the same "spending psychology" as insurance for 
these housewives. None of these commodities are necessary to the point of wanting to go out of the way to pay for them, but they seem to be equated with a feeling of uneasiness unless paid for. Messy hair for a housewife is unsettling, just as an unsent "oseibo" brings about a feeling of uneasiness that the other person will be offended and their relationship will suffer as a result. Under such conditions, it is possible to say that "oseibo" carries with it a very social and formal connotation recognized by everybody, unlike the personal connotation which is associated with birthday and Christmas presents.

(6) Feminine articles. This factor shows slightly different commodities depending upon the scale. Under the "necessary" scale, in particular, hair care products seem to be paid for from a different "psychology of spending" than the other feminine articles. It has traditionally been said that hair is a woman's pride. This symbolic value for hair care and make-up has not changed today.

(7) Going out and eating out. This is the name of the 11th factor under the "necessary" scale. Whether the heading "going out" is appropriate or not is difficult to say, but in this case, eating out was considered one sort of going out. It is also interesting to note that the coffee and cake bills, paid for out of the "going out" expense under "popularity" and "psychological grudging accompanying expenditure" scales, are separated under the "necessary" scale. Meals and coffeebreak foods are psychologically separated probably according to their necessity.

The above seven factors were common to all three scales, but since there are several others whose special properties cannot be ignored, they are briefly mentioned here.

\section{Other "spending psychologies".}

(1) Pocket money. This is the factor 1 under "psychological grudging accom- panying expenditure" scale, but was not found as a main factor in the previous survey. There seems to be no connection between the commodities except for the fact that they are cheap. In other words, it is possible to say that the important fact influencing the "psychological grudging accompanying expenditure" does not depend upon other properties of the commodities, but rather there is a stronger tendency to be influenced by the price itself.

(2) Raising the level of the standard of living. This is also a factor extracted only from the "psychological grudging accompanying expenditure " scale, but when its roles and functions are considered, it is a "moneybag" with an important meaning. This expenditure is classified between "expenses for daily necessities" and that "for a small luxury". That is, these commodities are not really necessary but are not really luxuries; they are those things which are given priority in purchasing, if there is a little extra to spare to make life more pleasant. The commodities included in this factor are: cassettetape recorder, microwave oven, central heating and air conditioning, although among these, the microwave oven and air conditioning are items which seem to be moving up to become a "necessity". In general, those commodities which are not yet widely distributed and considered a luxury in the initial selling phase, can be transformed into more popular commodities by price cuts. These cuts will be possible either by new technological improvements or advertising activities. These eventually become recognized as commodities required to raise the standard of living and, depending upon the product, may become a commodity ranked as a daily necessity. Under such conditions, the three factors such as "a small luxury ", " raising the level of the standard of living", and "daily necessity" are useful indexes in considering the process of changing consumer consciousness at a 
given period of time. As a concrete example, color television is a typical commodity showing rapid shift along these three scales. The disappearance of the car, as number 1 in the 1972 survey under the factor " raising the level of the standard of living ", from this survey and appearing as the factor "a small luxury" may be related to the recent energy crisis.

The above mentioned, "psychologies of spending" will be considered as items in a kind of psychological accounting book. Real housekeeping book items are too loyal to the superficial property of the commodity, so they are not too helpful in the analysis of purchasing activities. For example, the purchase of work clothes, children's play clothes, and a formal dress would probably all get listed under expenditures for "clothing". However, this loses important aspects of the psychological dimensions. This is the very reason why this survey was carried out to find "spending psychologies", which duly re- flects the trend of the consumer. As a result, important expenditures common to the three scales were found.

Follow-up surveys of the changes which occur over a period of time and the " intra-individual spending psychology for the same commodity", mentioned at the beginning, which differs according to individual and environmental variables, are left for further investigation.

\section{References}

Asahi Shinbun: Advertising Department. 1972 The observation and analysis of the "psychology of spending". Asahi Shinbun Co., Japan. (In Japanese)

Kojima, S. 1964 The study of consumer psychology. Japan Productivity Co. (In Japanese)

Kojima, S. 1972 The study of consumer psychology. New Edition. Japan Productivity Co. (In Japanese)

Tanaka, M.. \& Kitade, S. 1974 The psychology of spending. Diamond Co. (In Japanese)

(Received May 11, 1981 ; accepted March 13, 1982) 